\title{
Synthesis of a Spacer-Containing Tetrasaccharide Representing a Repeating Unit of the Capsular Polysaccharide of Streptococcus pneumoniae Type 6B
}

\author{
Mark-J. L. Thijssen, Koen M. Halkes, Johannis P. Kamerling, ${ }^{*}$ and Johannes F. G. Vliegenthart \\ Bijvoet Center, Department of Bio-Organic Chemistry, Utrecht University, P.O. Box 80.075, \\ NL-3508 TB Utrecht, The Netherlands
}

Abstract-The synthesis is reported of the spacer-containing tetrasaccharide $\alpha$-D-Galp-(1 $\rightarrow 3)-\alpha-\mathrm{D}-\mathrm{Glc} p-(1 \rightarrow 3)-\alpha-\mathrm{L}-\mathrm{Rh} a p-(1 \rightarrow 4)-$
D-RibOH- $\left(5 \rightarrow\right.$ phosphate $\rightarrow \mathrm{CH}_{2} \mathrm{CH}_{2} \mathrm{CH}_{2} \mathrm{NH}_{2}(1)$. using a $2+2$ block synthesis approach.

\section{Introduction}

Streptococcus pneumoniae is a gram-positive bacterium that can cause pneumonia, otitis media and meningitis in humans. To date, up to 85 different serotypes of this bacterium have been identified by their capsular polysaccharide. ${ }^{1}$ The current polyvalent vaccine Pneumovax ${ }^{\circledR} 23^{2}$ is constituted of the purified capsular polysaccharides of 23 serotypes of $S$. pneumoniae. Nevertheless, this vaccine is poorly immunogenic in persons of high risk groups such as children under the age of two, old persons, chronically ill people, and splenectomised patients. Since a polysaccharideinduced immune response does not evoke an immunological memory (TI-response), only short-term protection is provided. Moreover, the induction of tolerance is a severe problem. ${ }^{1}$ These disadvantages, especially the absence of long-term protection, may be overcome by the use of oligosaccharide-conjugate based vaccines.

In the framework of our studies towards oligosaccharideconjugate based vaccines against $S$. pneumoniae we became interested in one of the most virulent serotypes, namely type 6 , which can be divided into two crossreactive serotypes $6 \mathrm{~A}$ and $6 \mathrm{~B}$. In earlier reports ${ }^{3-5}$ we have described the synthesis of several fragments of the capsular polysaccharides of $S$. pneumoniae types $6 \mathrm{~A}$ and $6 \mathrm{~B}$, useful in immunological inhibition studies. These compounds did not contain a spacer, which is necessary to prepare neoglycoconjugates, essential for vaccination studies. Here, we report the synthesis of the spacercontaining retrasaccharide 1 representing a repeating unit of the capsular polysaccharide of $S$. pneumoniae type 6B (Fig. 1).

\section{Results and Discussion}

To establish a convenient and systematic synthesis of several different phosphate- and spacer-containing tetrasaccharide repeating units a strategy was developed, based on block synthesis. For the synthesis of the tetrasaccharide $O-\alpha$-D-galactopyranosyl- $(1 \rightarrow 3)-O$ $\alpha$-D-glucopyranosyl-( $1 \rightarrow 3)-O$ - $\alpha$-L -hamnopyranosyl- $(1 \rightarrow$ 4)-(5-O-[3-aminopropyl phosphate])-D-ribitol (1), the building blocks 5-O-allyl-1-O-benzoyl-2,3-di-O-benzyl-4$O$-( 2-O-a c e t yl -4-O-be n z y l- $\alpha$-I -rhamnopyranosyl)-D ribitol (11) and ethyl 2,4,6-tri-O-benzyl-3-O-(3,4,6-tri- $O$ benzyl-2- $O$-p-methoxybenzyl- $\alpha$ - D- galactopyranosyl)1- thio- $\beta$-D-gluco-pyranoside (26) were designed. In this case, the block synthesis approach is powerful because after protecting group manipulations both building blocks can easily be transformed into several larger target structures.

For the synthesis of disaccharide derivative 11 the rhamnose donor ethyl 2-O-acetyl-4-O-benzyl-3-O-monochloroacetyl-1-thio- $\alpha$-L-rhamnopyranoside (7) and the ribitol acceptor 5-O-allyl-1-O-benzoyl-2,3-di- $O$-benzyl-Dribitol (9) were prepared (Scheme I).

Ethyl 2,3,4-tri- $O$-acetyl-1-thio- $\alpha$-L-rhamnopyranoside (2) was deacetylated $(\rightarrow 3)$, and without purification converted in a one pot reaction, ${ }^{6}$ via orthoacetates 4 and 5 , into 6 ( $80 \%$ over 4 steps). Monochloroacetylation of HO-3, using monochloroacetyl chloride, then gave $7(83 \%)$. The previously described ${ }^{7} 5-O$ allyl-2,3-di-O-benzyl-D-ribitol (8) was selectively

$[\rightarrow 2)-\alpha-D-G a l p-(1 \rightarrow 3)-\alpha-D-G i c p-(1 \rightarrow 3)-\alpha-L-R h a p-(1 \rightarrow 4)-D-R i b O H-(5 \rightarrow \text { phosphate } \rightarrow]_{n}$

$\alpha$-D-Galp-(1 $\rightarrow 3)-\alpha$-D-Glcp-(1 $\rightarrow 3)$ - $\alpha$-L-Rhap-(1 $\rightarrow 4)-D-R i b O H-\left(5 \rightarrow\right.$ phosphate $\rightarrow\left(\mathrm{CH}_{2}\right){ }_{3} \mathrm{NH}_{2}$

Figure 1. The structure of (a) the capsular polysaccharide of $S$. pneumoniae type 6B and (b) the compound synthesised in this study (1). 


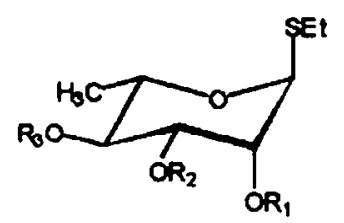

$$
\begin{aligned}
& 2 R_{1}=R_{2}=R_{3}=A C \\
& 3 R_{1}=R_{2}=R_{3}=H \\
& 4 R_{1}, R_{2}=C\left(O C H_{3}\right) C H_{3}, R_{3}=H \\
& 5 R_{1}, R_{2}=C\left(O C H_{3}\right) C H_{3}, R_{3}=B n \\
& 6 R_{1}=A C, R_{2}=H, R_{3}=B n \\
& 7 R_{1}=A C, R_{2}=m C A, R_{3}=B n
\end{aligned}
$$<smiles>[R9]C[C@H](O)[C@@H](O)[C@H](O)CO</smiles>

$8 \mathrm{R}=\mathrm{H}$

$9 \mathrm{R}=\mathrm{Bz}$

Scheme I. Ac $=$ acetyl, $\mathrm{mCA}=$ monochloroacetyl, $\mathrm{Bn}=$ benzyl, $\mathrm{Bz}=$ benzoyl.

benzoylated ${ }^{8}$ at HO-1 using 1-(benzoyloxy)benzotriazole to yield $9(91 \%)$ (Scheme I). Condensation of 9 with 7 in $\mathrm{CH}_{2} \mathrm{Cl}_{2}$-diethyl ether in the presence of $\mathrm{N}$-iodosuccinimide/triflic acid $^{9}$ gave disaccharide derivative $10(85 \%)$. De-monochloroacetylation of 10 using hydrazine dithiocarbonate ${ }^{10}$ yielded disaccharide acceptor 11 (87\%) (Scheme II).

For the synthesis of disaccharide derivative 26 the galactose moieties ethyl 3,4,6-tri-O-benzyl-2-O- $t$ butyldimethylsilyl-1-thio- $\beta$-D-galactopyranoside (18) and ethyl 3,4,6-tri- $O$-benzyl-2- $O$ - $p$-methoxybenzyl-1-thio- $\beta$-Dgalactopyranoside (19) were selected as possible donors, differing in protecting group at C-2 (Scheme III), but synthesised from the same precursor.

Bromide 12 was converted analogously to a published ${ }^{11}$ 4-step procedure via 13-15 into 16 (21\% from 12$)$. After deacetylation $(\rightarrow 17,99 \%)$ the HO-2 function was either $t$-butyldimethylsilylated or $p$-methoxybenzylated to give 18 and 19, respectively, in comparable yields ( $87 \%$ and $93 \%$ ).

The glucose acceptor ethyl 2,4,6-tri- $O$-acetyl-1-thio- $\beta$-Dglucopyranoside (22) (Scheme IV) was prepared from compound 20 by hydrogenolysis in the presence of $\mathrm{Pd} / \mathrm{C}$ $(\rightarrow 21,85 \%)$, followed by thioylation using ethanethiol and tin(IV) chloride (62\%). It should be noted that conversion of $\mathbf{2 0}$ into 22 , via a route comprising thioglycoside preparation followed by debenzylation, was not successful with respect to the hydrogenolysis.

Then, the 'disarmed'12 glucose acceptor 22 was glycosylated (Scheme V) with the 'armed' galactose donor 18 at $-60{ }^{\circ} \mathrm{C}$ in 1:5 1,2-dichloroethane:diethyl ether using iodonium dicollidineperchlorate (IDCP) as a promoter, yielding disaccharide derivative $23(49 \%)$. Fortunately, coupling of glucose acceptor 22 with galactose donor 19 in 1:5 1,2-dichloroethane:diethyl ether using IDCP, gave 24 in a much higher yield (83 $\%)$. Deacetylation of $24(\rightarrow 25$, quantitative) and subsequent benzylation afforded disaccharide derivative $26(83 \%)$, which was used as a donor in the block synthesis of the target tetrasaccharide 1 .

To obtain the title tetrasaccharide 1 a series of coupling reactions were carried out between acceptor 11 and donor 26 (Scheme VI). In the presence of IDCP tetrasaccharide derivative 31 was obtained in a yield of $67 \%$, but with little stereoselectivity $(\alpha / \beta$-ratio of $1: 1)$. When methyl triflate was used as a promoter, repeated attempts in diethyl ether led to low yields $(29 \%)$ of 31 , with an unexpected $\alpha / \beta$-ratio of $1: 2$, and a substantial amount of the donor-derived glycal 32.

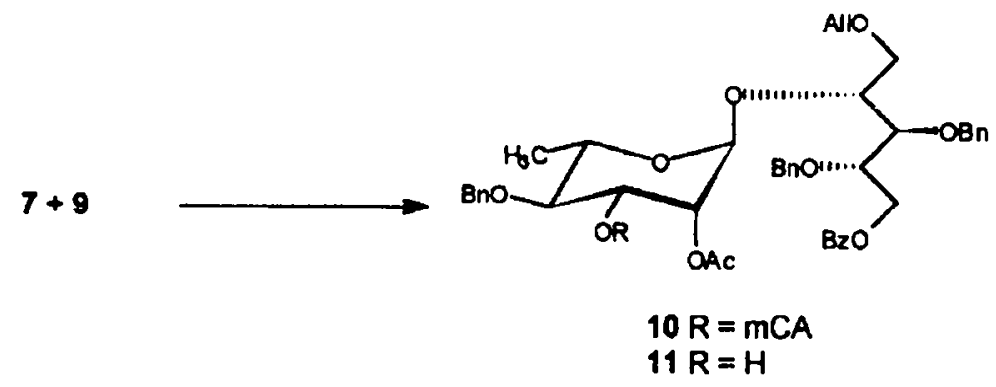

Scheme II. All = allyl.

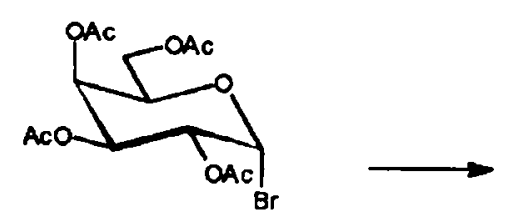

12
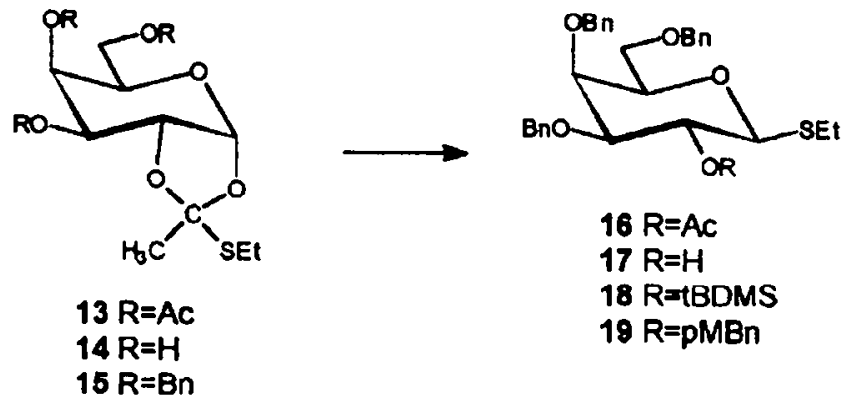

Scheme III. tBDMS $=t$-butyldimethylsilyl, $\mathrm{SEt}=$ thioethyl, $\mathrm{pMBn}=p$-methoxybenzyl. 


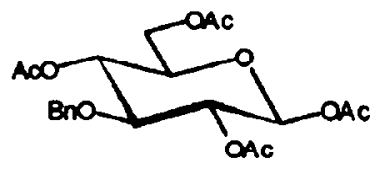

20

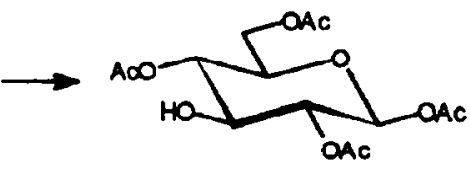

21

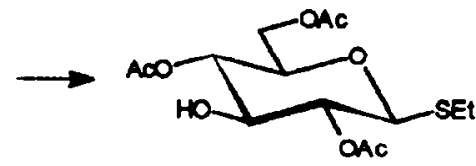

22

Scheme IV.

Scheme $\mathbf{V}$.

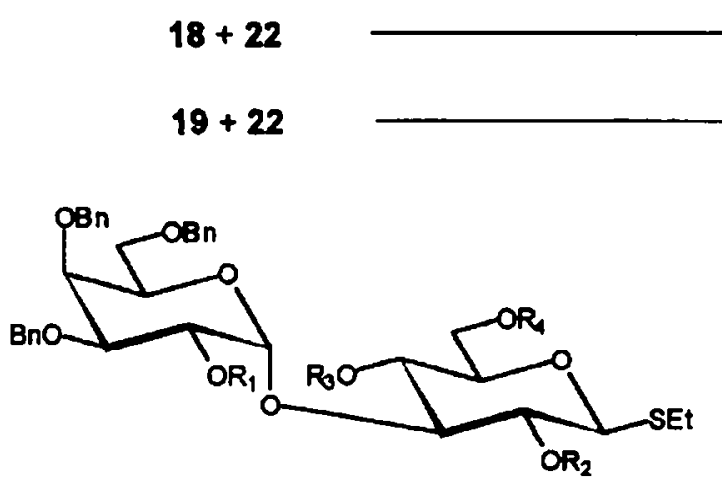

$23 R_{1}=t B D M S, R_{2}=R_{3}=R_{4}=A C$

$24 R_{1}=p M B n, R_{2}=R_{3}=R_{4}=A C$

$25 R_{1}=p M B n, R_{2}=R_{3}=R_{4}=H$

$26 R_{1}=p M B n, R_{2}=R_{3}=R_{4}=B n$

$27 R_{1}=p M B n, R_{2}=H, R_{3}, R_{4}=C_{1} C_{6} H_{5}$

$28 R_{1}=p M B n, R_{2}=B n, R_{3}, R_{4}=C C_{6} H_{5}$

$29 R_{1}=p M B n, R_{2}=B n, R_{3}=R_{4}=H$

$30 R_{1}=p M B n, R_{2}=B n, R_{3}=R_{4}=A C$

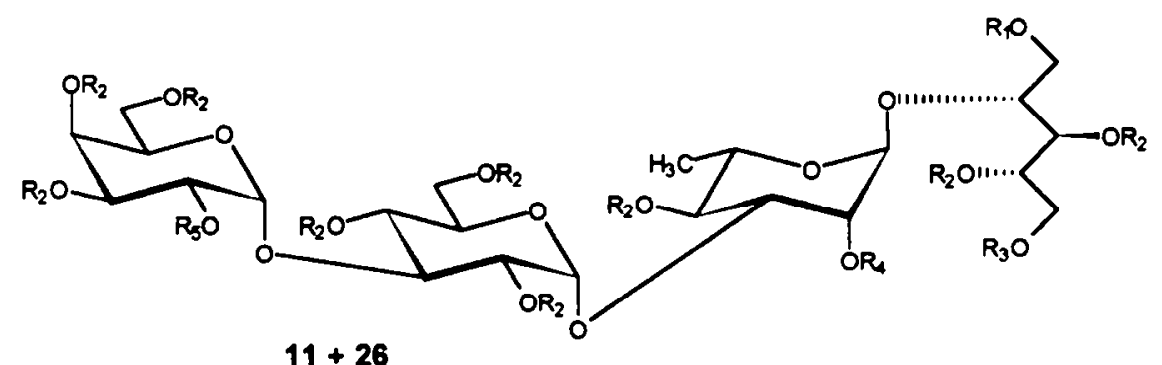

$11+26$

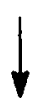

$31 \alpha R_{1}=A l l, R_{2}=B n, R_{3}=B z, R_{4}=A C, R_{5}=p M B n$

$33 R_{1}=H, R_{2}=B n, R_{3}=B z, R_{4}=A c, R_{5}=p M B n$

$34 R_{1}=\prod_{N}$

$35 R_{1}=\overbrace{l_{H}}^{\infty}$

$36 R_{1}=\overbrace{\mathrm{NHZ}}^{l_{\mathrm{O}}}, \mathrm{R}_{2}=B n, R_{3}=R_{4}=H, R_{5}=p M B n$

$1 R_{1}=\overbrace{I_{\mathrm{O}}}^{l_{1}}, R_{2}=R_{3}=R_{4}=R_{5}=H$

Scheme VI. $\mathrm{Z}$ = benzyloxycarbonyl.

To overcome the low stereoselectivity of the glycosylation reaction between 11 and 26 , less reactive variants of 26 were prepared, namely 28 and 30 (Scheme V). To this end, compound 24 was deacetylated $(\rightarrow 25)$, and without purification benzylidenated using $\alpha, \alpha$-dimethoxytoluene to yield $\mathbf{2 7}$ (60\% over 2 steps). After benzylation of the HO-2 group $(\rightarrow 28,89 \%)$, the benzylidene function was 
removed with aq. trifluoroacetic acid in $\mathrm{CH}_{2} \mathrm{Cl}_{2}$, affording $29(41 \%)$ and a compound without $p$ methoxybenzyl function $(9 \%)$. Subsequent acetylation of 29 gave $30(73 \%)$. Test reactions with 28 as glycosyl donor using IDCP or methyl triflate as promoters were not successful, possibly due to the presence of the benzylidene protective group. It is known ${ }^{13}$ that the reactivity of a donor molecule is reduced by the presence of a 4,6-dioxane ring. 4,6-Dioxane ring systems give a donor a more rigid structure, thereby hampering the formation of an anomeric oxonium cation in a glycosyl donor during activation. Also a test reaction with 30 as glycosyl donor using methyl triflate as a promoter in diethyl ether $\left(-40^{\circ} \mathrm{C} \rightarrow+20^{\circ} \mathrm{C}\right.$ in $\left.5 \mathrm{~h}\right)$ failed. A complex mixture of products was found without the desired compound.

The $\alpha / \beta$-mixture of 31 could only be separated after deallylation (Wilkinson catalyst) of the ribitol unit $(\rightarrow 33,37 \%)$ (Scheme VI). Phosphorylation of HO-5 of the ribitol unit in 33 using 3-N-(benzyloxycarbonyl)aminopropyl phosphonate with pivaloyl chloride in pyridine $^{14}$ gave the spacer-tetrasaccharide-phosphonate diester $34(53 \%)$. Oxidation of 34 with iodine in pyridine-water, effecting the conversion of the phosphonate group into a phosphate group, gave 35 (65 $\%)$. Finally, deacetylation/debenzoylation in methanolammonia $(\rightarrow 36)$ followed, without further purification, by hydrogenolysis to remove benzyl, $p$-methoxybenzyl and benzyloxycarbonyl functions, and purification on Bio-Gel P-2 gave the title oligosaccharide 1 ( $75 \%$ over the last 2 steps).

The conjugation of 1 to carrier proteins and the results of immunological tests with this conjugate will be reported elsewhere.

\section{Experimental}

\section{General methods}

${ }^{1} \mathrm{H}$ NMR spectra (300 and $500 \mathrm{MHz}$ ) were recorded at $25{ }^{\circ} \mathrm{C}$ with Bruker AC 300 and Bruker AM 500 spectrometers. Chemical shifts $(\delta)$ are given in ppm relative to the signal for internal $\mathrm{Me}_{4} \mathrm{Si}\left(\mathrm{CDCl}_{3}\right)$ or internal sodium 4,4-dimethyl-4-silapentane-1-sulfonate $\left(\mathrm{D}_{2} \mathrm{O}\right.$; indirectly to internal acetone, $\delta$ 2.225). Column chromatography was performed on Kieselgel 60 (Merck, $<230$ mesh or $70-230$ mesh) and fractions were monitored by TLC on Kicselgel $60 F_{254}$ (Merck), using detection with UV light and then charring with aq. $50 \%$ sulfuric acid. Optical rotations were measured at $20{ }^{\circ} \mathrm{C}$ for solutions in $\mathrm{CHCl}_{3}$ (unless stated otherwise) with a Perkin-Elmer 241 polarimeter, using a $10-\mathrm{cm} 1-\mathrm{mL}$ cell. Melting points (uncorrected) were determined with a Kofler apparatus. Evaporations were conducted under reduced pressure at $40{ }^{\circ} \mathrm{C}$ (bath). Reactions were performed under dry conditions using an atmosphere of nitrogen or argon. All solvents were distilled from appropriate drying agents.
Ethyl 2-O-acetyl-4-O-benzyl-1-thio- $\alpha$-L-rhamnopyranoside (6)

To a solution of ethyl 2,3,4-tri- $O$-acetyl-1-thio- $\alpha$-L rhamnopyranoside ${ }^{15}(2 ; 4.0 \mathrm{~g}, 12 \mathrm{mmol})$ in methanol $(20 \mathrm{~mL})$ was added sodium methoxide $(\mathrm{pH} 10)$. When TLC (9:1, $\mathrm{CH}_{2} \mathrm{Cl}_{2}$ :methanol) showed the deacetylation to be completed (10 min), the mixture was concentrated and co-concentrated with $\mathrm{CH}_{2} \mathrm{Cl}_{2}$. Crude 3 was dissolved in toluene $(30 \mathrm{~mL})$, and $\mathrm{CH}_{2} \mathrm{Cl}_{2}(5 \mathrm{~mL}), p$ toluenesulfonic acid $(200 \mathrm{mg})$, and trimethyl orthoacetate $(6.1 \mathrm{~mL}, 48 \mathrm{mmol})$ were added. After 45 min, TLC $\left(9: 1, \mathrm{CH}_{2} \mathrm{Cl}_{2}\right.$ : methanol) showed a complete conversion of 3 into $4\left(R_{f} 0.70\right)$. Then, DMF (30 mL) and sodium hydride $(1.0 \mathrm{~g}, 42 \mathrm{mmol})$ were added, and the mixture was stirred until the evolution of gas stopped. Benzyl bromide was added in two portions (1.5 $\mathrm{mL}, 13 \mathrm{mmol}$; after $90 \mathrm{~min}: 2 \mathrm{~mL}, 17 \mathrm{mmol}$ ), and the mixture was stirred for $2.5 \mathrm{~h}$, when TLC $(6: 4$, hexane:FtOAc) showed the benzylation to be completed $\left(5, R_{f} 0.89\right)$. After destroying the excess of sodium hydride with methanol, the mixture was diluted with EtOAc, washed with water $(3 \times)$, and concentrated. The residue was dissolved in aq. $80 \%$ acetic acid $(20 \mathrm{~mL})$, and the solution was stirred for $5 \mathrm{~min}$, and coconcentrated with toluene $(3 x)$, ethanol $(3 x)$, and $\mathrm{CH}_{2} \mathrm{Cl}_{2}(3 \times)$. Column chromatography $(75: 25$, hexane:EtOAc) of the residue yielded $6(3.27 \mathrm{~g}, 80 \%)$ as a syrup, $[\alpha]_{D}-120^{\circ}(\mathrm{c} 1)$. ' $\mathrm{H}$ NMR $\left(\mathrm{CDCl}_{3}\right) \delta 7.37-$ $7.26(\mathrm{~m}, 5 \mathrm{H}, \mathrm{Ph}), 4.822$ and $4.717(2 \mathrm{~d}$, each $1 \mathrm{H}$, $\left.\mathrm{OC}_{2} \mathrm{Ph}\right), 4.088(\mathrm{~m}, 1 \mathrm{H}, \mathrm{H}-5), 4.051(\mathrm{dd}, 1 \mathrm{H}, \mathrm{H}-3)$, 3.382 (t, 1H, H-4), 2.68-2.54 (m, 2H, SCH $\mathrm{CH}_{3}$ ), 2.160 (s, 3H, Ac), 1.356 (d, 3H, $3 \mathrm{H}-6), 1.276(\mathrm{t}, 3 \mathrm{H}$, $\left.\mathrm{SCH}_{2} \mathrm{CH}_{3}\right) ; J_{2,3}=3.1, J_{3,4}=9.4, J_{4,5}=9.4, J_{5,6}=6.2$ $\mathrm{Hz}$; Anal. calcd for $\mathrm{C}_{17} \mathrm{H}_{24} \mathrm{O}_{5} \mathrm{~S}: \mathrm{C}, 59.98 ; \mathrm{H}, 7.11 \%$; found: $\mathrm{C}, 59.69 ; \mathrm{H}, 7.06 \%$.

Ethyl 2-O-acetyl-4-O-benzyl-3-O-monochloroacetyl-1thio- $\alpha$-L-rhamnopyranoside (7)

To a stirred solution of $6(14.2 \mathrm{~g}, 41.7 \mathrm{mmol})$ in $\mathrm{CH}_{2} \mathrm{Cl}_{2}$ $(125 \mathrm{~mL})$ and pyridine $(4.05 \mathrm{~mL})$ at $0{ }^{\circ} \mathrm{C}$ was added dropwise in $1 \mathrm{~h}$ a solution of monochloroacetyl chloride (3.34 $\mathrm{mL}, 42 \mathrm{mmol})$ in $\mathrm{CH}_{2} \mathrm{Cl}_{2}(40 \mathrm{~mL})$. After additional stirring for $90 \mathrm{~min}$, TLC (7:3, hexane:EtOAc) showed the formation of $7\left(R_{f} 0.55\right)$. The mixture was diluted with $\mathrm{CH}_{2} \mathrm{Cl}_{2}$, washed with water $(3 \times)$, dried $\left(\mathrm{MgSO}_{4}\right.$ ), filtered, and concentrated. Column chromatography $(9: 1$, toluene:EtOAc) of the residue gave $7(14.02 \mathrm{~g}, 83 \%)$ as a white solid, $\mathrm{mp} 61{ }^{\circ} \mathrm{C} ;[\alpha]_{\mathrm{D}}$ $-98^{\circ}$ (c 1). ${ }^{1} \mathrm{H}$ NMR $\left(\mathrm{CDCl}_{3}\right) \delta 7.34-7.28(\mathrm{~m}, 5 \mathrm{H}, \mathrm{Ph})$, $5.375(\mathrm{dd}, 1 \mathrm{H}, \mathrm{H}-2), 5.290(\mathrm{dd}, 1 \mathrm{H}, \mathrm{H}-3), 5.116(\mathrm{~d}, 1 \mathrm{H}$, $\mathrm{H}-1$ ), 4.709 and 4.659 (2d, each $1 \mathrm{H}, \mathrm{OCH}_{2} \mathrm{Ph}$ ), 4.199 (dq, $1 \mathrm{H}, \mathrm{H}-5), 3.912$ and 3.847 (2d, each $1 \mathrm{H}$, $\left.\mathrm{COC}_{2} \mathrm{Cl}\right), 3.569(\mathrm{t}, 1 \mathrm{H}, \mathrm{H}-4), 2.69-2.55(\mathrm{~m}, 2 \mathrm{H}$, $\left.\mathrm{SC} \mathrm{H}_{2} \mathrm{CH}_{3}\right), 2.146(\mathrm{~s}, 3 \mathrm{H}, \mathrm{Ac}), 1.362$ (d, $\left.3 \mathrm{H}, 3 \mathrm{H}-6\right)$, $1.283\left(\mathrm{t}, 3 \mathrm{H}, \mathrm{SCH}_{2} \mathrm{CH}_{3}\right) ; J_{1,2}=1.6, J_{2.3}=3.4, J_{3,4}=$ 9.6, $J_{4,5}=9.6, J_{5,6}=6.2 \mathrm{~Hz}$; Anal. calcd for $\mathrm{C}_{19} \mathrm{H}_{25} \mathrm{ClO}_{6} \mathrm{~S}: \mathrm{C}, 54.74 ; \mathrm{H}, 6.04 \%$; found: $\mathrm{C}, 54.68 ; \mathrm{H}$, $6.16 \%$ 
5-O-Allyl-1-O-benzoyl-2,3-di-O-benzyl-D-ribitol (9)

A solution of 5 - $O$-allyl-2,3-di- $O$-benzyl-D-ribitol ${ }^{7}(8 ; 2.5$ $\mathrm{g}, 6.7 \mathrm{mmol})$ and 1 -(benzoyloxy)-benzotriazole $(1.8 \mathrm{~g}$, $7.5 \mathrm{mmol})$ in $\mathrm{CH}_{2} \mathrm{Cl}_{2}(33.5 \mathrm{~mL})$ and triethylamine $(1.1$ $\mathrm{mL})$ was stirred overnight. Then TLC $(8: 2$, hexane:EtOAc) showed the exclusive formation of $9\left(R_{f}\right.$ 0.38 ), and the mixture was diluted with $\mathrm{CH}_{2} \mathrm{Cl}_{2}$, washed with aq. $10 \% \mathrm{NaHCO}_{3}$, brine and water, dried $\left(\mathrm{MgSO}_{4}\right)$, filtered, and concentrated. Column chromatography $(85: 15$, hexane:EtOAc) of the residue gave $9(3.2 \mathrm{~g}, 91 \%)$ as a syrup, $[\alpha]_{\mathrm{D}}-39^{\circ}$ (c 1$) .{ }^{1} \mathrm{H}$ NMR $\left(\mathrm{CDCl}_{3}\right) \delta 8.20-7.22(\mathrm{~m}, 15 \mathrm{H}, 3 \mathrm{Ph}), 5.882(\mathrm{~m}$, $\left.1 \mathrm{H}, \mathrm{OCH}_{2} \mathrm{CH}=\mathrm{CH}_{2}\right), 5.247$ and $5.166(2 \mathrm{dq}$, each $1 \mathrm{H}$, $\left.\mathrm{OCH}_{2} \mathrm{CH}=\mathrm{CH}_{2}\right), 4.80-4.64\left(\mathrm{~m}, 4 \mathrm{H}, 2 \mathrm{OCH}_{2} \mathrm{Ph}\right), 4.762$ (dd, 1H, H-1a), 4.514 (dd, 1H, H-1b), 4.106 (ddd, 1H, $\mathrm{H}-2), 4.036$ (ddd, $1 \mathrm{H}, \mathrm{H}-4), 3.98-3.95(\mathrm{~m}, 2 \mathrm{H}$, $\mathrm{OCH}_{2} \mathrm{CH}=\mathrm{CH}_{2}$ ), 3.831 (dd, 1H, H-3), 3.597 (dd, 1H, H$5 \mathrm{a}), 3.551$ (dd, $1 \mathrm{H}, \mathrm{H}-5 \mathrm{~b}) ; J_{1 \mathrm{a}, 1 \mathrm{~b}}=12.1, J_{1 \mathrm{a}, 2}=3.2, J_{1 \mathrm{~b}, 2}$ $=6.1, J_{2,3}=6.9, J_{3,4}=4.2, J_{4,5 \mathrm{a}}=3.5, J_{4,5 \mathrm{~b}}=5.8, J_{5 \mathrm{a}, 5 \mathrm{~b}}$ $=9.7 \mathrm{~Hz}$.

5-O-Allyl-1-O-benzoyl-2,3-di-O-benzyl-4-O-(2-0-acetyl-4O-be nzyl-3-O-monochloroacetyl- $\alpha$-L-rhamnopyranosyl)D-ribitol (10)

A mixture of $9(1.04 \mathrm{~g}, 2.18 \mathrm{mmol}), 7(1.00 \mathrm{~g}, 2.40$ mmol) and $4 \AA$ molecular sieves $(1.7 \mathrm{~g})$ in $\mathrm{CH}_{2} \mathrm{Cl}_{2}$ $(6.45 \mathrm{~mL})$ was stirred for $30 \mathrm{~min}$. Then, at $0{ }^{\circ} \mathrm{C}$ a mixture of $N$-iodosuccinimide $(0.57 \mathrm{~g}, 2.4 \mathrm{mmol})$ and triflic acid $(19 \mu \mathrm{L}, 0.21 \mu \mathrm{mol})$ in $1: 1 \mathrm{CH}_{2} \mathrm{Cl}_{2}$ :diethyl ether $(19.5 \mathrm{~mL})$ was added. After $1 \mathrm{~min}$, TLC $(9: 1$, toluene:EtOAc) showed the appearance of a single product $\left(10, R_{f} 0.36\right)$, and the mixture was neutralised with triethylamine, filtered over Celite, diluted with $\mathrm{CH}_{2} \mathrm{Cl}_{2}$, washed with aq. $10 \%$ sodium thiosulfate $(2 \times)$ and water $(3 \times)$, dried $\left(\mathrm{MgSO}_{4}\right)$, filtered, and concentrated. Column chromatography $(9: 1$, toluene:EtOAc) of the residue afforded $10(1.55 \mathrm{~g}, 85$ \%) as a syrup, $[\alpha]_{\mathrm{D}}-46^{\circ}$ (c 1$) .{ }^{1} \mathrm{H}$ NMR $\left(\mathrm{CDCl}_{3}\right) \delta$ 7.95-7.31 (m, 20H, $4 \mathrm{Ph}), 5.836(\mathrm{~m}, 1 \mathrm{H}$, $\left.\mathrm{OCH}_{2} \mathrm{CH}=\mathrm{CH}_{2}\right), 5.21-5.09\left(\mathrm{~m}, 2 \mathrm{H}, \mathrm{OCH}_{2} \mathrm{CH}=\mathrm{CH} H_{2}\right)$, 5.141 (d, 1H, H-1'), 4.759 (dd, 1H, H-1b), 4.455 (dd, 1H, H-la), 4.313 (m, 1H, H-5'), 3.818 (dd, 1H, H-3), 3.629 (d, 2H, H-5a,5b), $3.527\left(\mathrm{t}, 1 \mathrm{H}, \mathrm{H}-4^{\prime}\right), 2.146(\mathrm{~s}, 3 \mathrm{H}, \mathrm{Ac})$, $1.163\left(\mathrm{~d}, 3 \mathrm{H}, 3 \mathrm{H}-6^{\prime}\right) ; J_{1 \mathrm{a}, 1 \mathrm{~b}}=12.0, J_{1 \mathrm{a}, 2}=4.9, J_{1 \mathrm{~b}, 2}=$ $6.8, J_{2,3}=7.2, J_{3,4}=2.9, J_{4,5 \mathrm{a}}=5.3, J_{4,5 \mathrm{~b}}=5.3, J_{1^{\prime}, 2^{\prime}}=$ $1.4, J_{3^{\prime}, 4^{\prime}}=10.0, J_{4^{\prime}, 5^{\prime}}=10.0, J_{5^{\prime}, 6^{\prime}}=6.2 \mathrm{~Hz}$; Anal. calcd for $\mathrm{C}_{46} \mathrm{H}_{51} \mathrm{ClO}_{12}: \mathrm{C}, 66.46 ; \mathrm{H}, 6.18 \%$; found: $\mathrm{C}, 66.34$; $\mathrm{H}, 6.08 \%$.

5-0-Allyl-1-O-benzoyl-2,3-di-O-benzyl-4-O-(2-O-acetyl-4O-benzyl- $\alpha$-L-rhamnopyranosyl)-D-ribitol (11)

To a solution of $10(705 \mathrm{mg}, 0.82 \mathrm{mmol})$ in $1: 3$ acetic acid:2,6-lutidine $(17 \mathrm{~mL})$ was added a freshly prepared hydrazine dithiocarbonate solution ${ }^{10}(4 \mathrm{~mL})$. TLC (7:3, hexane:EtOAc, $R_{f} 0.22$ ) showed the demonochloroacetylation to be complete in $15 \mathrm{~min}$. Then, the mixture was diluted with $\mathrm{CH}_{2} \mathrm{Cl}_{2}$, and washed with water $(3 \times)$, dried $\left(\mathrm{MgSO}_{4}\right)$, filtered, and concentrated. Column chromatography $(7: 3$, hexane:EtOAc) of the residue gave $11(539 \mathrm{mg}, 87 \%)$ as a syrup, $[\alpha]_{\mathrm{D}}-47^{\circ}$ (c 1). ${ }^{1} \mathrm{H}$ NMR $\left(\mathrm{CDCl}_{3}\right) \delta 8.05-7.33(\mathrm{~m}, 20 \mathrm{H}, 4 \mathrm{Ph}), 5.861(\mathrm{~m}$, $\left.1 \mathrm{H}, \quad \mathrm{OCH}_{2} \mathrm{CH}=\mathrm{CH}_{2}\right), \quad 5.24-5.10 \quad(\mathrm{~m}, \quad 2 \mathrm{H}$, $\mathrm{OCH}_{2} \mathrm{CH}=\mathrm{CH}_{2}$ ), 5.164 (dd, 1H, H-2'), 5.099 (d, 1H, H$\left.\mathrm{I}^{\prime}\right), 4.761,4.731,4.714,4.663,4.635$, and $4.585(6 \mathrm{~d}$, each $1 \mathrm{H}, 3 \mathrm{OCH}_{2} \mathrm{Ph}$ ), 4.750 (dd, $\left.1 \mathrm{H}, \mathrm{H}-1 \mathrm{a}\right), 4.449$ (dd, $1 \mathrm{H}, \mathrm{H}-1 \mathrm{~b}), 4.251$ (dq, 1H, H-2), 4.041 (dd, 1H, H-3'), $3.324\left(\mathrm{t}, 1 \mathrm{H}, \mathrm{H}-4^{\prime}\right), 1.160\left(\mathrm{~d}, 3 \mathrm{H}, 3 \mathrm{H}-6^{\prime}\right) ; J_{1 \mathrm{a}, 1 \mathrm{~b}}=12.0$, $J_{1 \mathrm{a}, 2}=2.5, J_{1 \mathrm{~b}, 2}=5.2, J_{1^{\prime}, 2^{\prime}}=1.7, J_{2^{\prime}, 3^{\prime}}=3.4, J_{3^{\prime}, 4^{\prime}}=9.5$, $J_{4^{\prime}, 5^{\prime}}=9.5, J_{5^{\prime}, 6^{\prime}}=6.2 \mathrm{~Hz}$; Anal. calcd for $\mathrm{C}_{44} \mathrm{H}_{50} \mathrm{O}_{11} \cdot 1 / 2 \mathrm{H}_{2} \mathrm{O}: \mathrm{C}, 69.18 ; \mathrm{H}, 6.73 \%$; found: $\mathrm{C}, 69.29$; $\mathrm{H}, 6.80 \%$.

Ethyl 2-0-acetyl-3,4,6-tri-O-benzyl-1-thio- $\alpha$-D-galactopyranoside (16)

To a solution of 2,3,4,6-tetra- $O$-acetyl- $\alpha$-D-galactopyranosyl bromide $(12 ; 18.9 \mathrm{~g}, 46 \mathrm{mmol})$ in nitromethane $(40 \mathrm{~mL})$ were added tetraethylammonium bromide $(0.96 \mathrm{~g}, 4.6 \mathrm{mmol}), 2,6$-lutidine $(7.8 \mathrm{~mL}, 67$ $\mathrm{mmol})$, and ethanethiol (13.6 $\mathrm{mL}, 184 \mathrm{mmol})$. TLC (95:5, $\mathrm{CH}_{2} \mathrm{Cl}_{2}$ :EtOAc) showed the conversion of $12\left(R_{f}\right.$ $0.57)$ into $13\left(R_{f} 0.81\right)$ after $24 \mathrm{~h}$. Then, the mixture was diluted with EtOAc, washed with aq. $10 \% \mathrm{NaHCO}_{3}(2$ $x)$ and water, dried $\left(\mathrm{MgSO}_{4}\right)$, filtered, and concentrated. Column chromatography (69:30:1, hexane:EtOAc:triethylamine) of the residue yielded 13 as a syrup. To a solution of 13 in methanol ( $200 \mathrm{~mL}$ ) was added sodium methoxide ( $\mathrm{pH} \mathrm{10}$ ), and the solution was stirred for $21 \mathrm{~h}$. When TLC (85:15, $\mathrm{CH}_{2} \mathrm{Cl}_{2}$ : acetone) showed the disappearance of $13\left(\rightarrow 14, R_{f}\right.$ 0.12 ), the solution was concentrated without neutralisation, co-concentrated twice with $\mathrm{CH}_{2} \mathrm{Cl}_{2}$, and dried in vacuo. A solution of crude 14 and benzyl bromide ( $16.6 \mathrm{~mL}, 140 \mathrm{mmol}$ ) in DMF $(60 \mathrm{~mL})$ was added dropwise to a cooled $\left(0^{\circ} \mathrm{C}\right)$ suspension of sodium hydride $(4.0 \mathrm{~g}, 167 \mathrm{mmol})$ in DMF $(40 \mathrm{~mL})$. TLC $(8: 2$, hexane:EtOAc) showed the formation of $15\left(R_{f} 0.69\right)$ to be completed in $24 \mathrm{~h}$. After destroying the excess of sodium hydride with methanol, the mixture was diluted with $\mathrm{EtOAc}$, washed with water $(3 \times)$, dried $\left(\mathrm{MgSO}_{4}\right)$, filtered, and concentrated to yield $15(22.3 \mathrm{~g})$ as a yellow syrup.

To a solution of crude $15(13.3 \mathrm{~g})$ in $\mathrm{CH}_{2} \mathrm{Cl}_{2}(50 \mathrm{~mL})$ was added $4 \AA$ molecular sieves $(4 \mathrm{~g})$, and the mixture was stirred for $30 \mathrm{~min}$. The solution was cooled $\left(0^{\circ} \mathrm{C}\right)$, and trimethylsilyl triflate $(0.50 \mathrm{~mL}, 2.7 \mathrm{mmol})$ was added. TLC (6:4, hexane:EtOAc) showed a complete conversion of 15 into $16\left(R_{f} 0.55\right)$ in $2.5 \mathrm{~h}$. Then, the mixture was neutralised with triethylamine, diluted with $\mathrm{CH}_{2} \mathrm{Cl}_{2}$, washed with water $(2 \times)$, dried $\left(\mathrm{MgSO}_{4}\right)$, filtered, and concentrated. Column chromatography (85:15, hexane:EtOAc) of the residue gave $16(5.2 \mathrm{~g}, 21$ $\%$ from 12) as a white solid, $m p 56^{\circ} \mathrm{C} ;[\alpha]_{D}-1{ }^{\circ}$ (c 1$)$. ${ }^{1} \mathrm{H} \mathrm{NMR}\left(\mathrm{CDCl}_{3}\right) \delta 7.32-7.28(\mathrm{~m}, 15 \mathrm{H}, 3 \mathrm{Ph}), 5.419(\mathrm{t}$, $1 \mathrm{H}, \mathrm{H}-2), 4.940$ and 4.533 (2d, each $1 \mathrm{H}, \mathrm{OCH}_{2} \mathrm{Ph}$ ), 4.671 and $4.574\left(2 \mathrm{~d}\right.$, each $\left.1 \mathrm{H}, \mathrm{OCH}_{2} \mathrm{Ph}\right), 4.460$ and 
$4.410\left(2 \mathrm{~d}\right.$, each $\left.1 \mathrm{H}, \mathrm{OCH}_{2} \mathrm{Ph}\right), 4.330(\mathrm{~d}, 1 \mathrm{H}, \mathrm{H}-1), 3.990$ (d, 1H, H-4), 3.537 (dd, 1H, H-3), 2.76-2.62 (m, 2H, $\mathrm{SCH}_{2} \mathrm{CH}_{3}$ ), 2.031 (s, 3H, Ac), 1.223 (t, $3 \mathrm{H}, \mathrm{SCH}_{2} \mathrm{CH}_{3}$ ); $J_{1,2}=9.8, J_{2,3}=9.8, J_{3,4}=2.8, J_{4,5}=0 \mathrm{~Hz}$; Anal. calcd for $\mathrm{C}_{31} \mathrm{H}_{36} \mathrm{O}_{6} \mathrm{~S}: \mathrm{C}, 69.38 ; \mathrm{H}, 6.76 \%$; found: $\mathrm{C}, 69.51 ; \mathrm{H}$, $6.79 \%$.

\section{Ethyl 3,4,6-tri-O-benzyl-1-thio- $\beta$-D-galactopyranoside (17)}

To a solution of $16(649 \mathrm{mg}, 1.31 \mathrm{mmol})$ in methanol (5 $\mathrm{mL}$ ) was added sodium methoxide ( $\mathrm{pH} 10)$. After $24 \mathrm{~h}$, TLC (6:4, hexane:EtOAc) showed a complete conversion into $17\left(R_{f} 0.49\right)$. The mixture was neutralised with Dowex-50 $\left(\mathrm{H}^{+}\right)$resin, filtered, concentrated, and co-concentrated with $\mathrm{CH}_{2} \mathrm{Cl}_{2}(2 \times)$ and ethanol, to give $17(593 \mathrm{mg}, 99 \%)$ as a white crystalline solid, mp $73^{\circ} \mathrm{C} ;[\alpha]_{\mathrm{D}}-7^{\circ}$ (c 1). ${ }^{1} \mathrm{H}$ NMR $\left(\mathrm{CDCl}_{3}\right) \delta 7.31-7.26(\mathrm{~m}, 15 \mathrm{H}, 3 \mathrm{Ph}), 4.894,4.593$, $4.744,4.686,4.478$, and 4.422 (6d, each $1 \mathrm{H}, 3$ $\left.\mathrm{OCH}_{2} \mathrm{Ph}\right), 4.313(\mathrm{~d}, 1 \mathrm{H}, \mathrm{H}-1), 3.978(\mathrm{t}, 1 \mathrm{H}, \mathrm{H}-2), 3.972$ (d, 1H, H-4), 3.447 (dd, 1H, H-3), 2.76-2.67 (m, 2H, $\left.\mathrm{SC} \mathrm{H}_{2} \mathrm{CH}_{3}\right), 1.286\left(\mathrm{t}, 3 \mathrm{H}, \mathrm{SCH}_{2} \mathrm{CH}_{3}\right) ; J_{1,2}=9.6, J_{2,3}=$ 9.5, $J_{3,4}=2.8, J_{4,5}=0 \mathrm{~Hz}$; Anal. calcd for $\mathrm{C}_{29} \mathrm{H}_{34} \mathrm{O}_{5} \mathrm{~S}$ : C, $70.42 ; \mathrm{H}, 6.93 \%$; found: C, $70.31 ; \mathrm{H}, 7.04 \%$.

Ethyl 3,4,6-tri-O-benzyl-2-O-t-butyldimethylsilyl-1-thio- $\beta$ D-galactopyranoside (18)

To a solution of $17(500 \mathrm{mg}, 0.82 \mathrm{mmol})$ in acetonitrile $(5 \mathrm{~mL})$ were added $t$-butyldimethylsilyl chloride $(0.20 \mathrm{~g}$, $1.3 \mathrm{mmol})$ and diazabicyclo[2.2.2] octane $(0.12 \mathrm{~g}, 1.1$ mmol). After $2 \mathrm{~h}$, TLC (95:5, $\mathrm{CH}_{2} \mathrm{Cl}_{2}$ :acetone) showed the disappearance of 17 and appearance of $18\left(R_{f} 0.78\right)$. The mixture was diluted with $\mathrm{CH}_{2} \mathrm{Cl}_{2}$, washed with water $(3 \times)$, dried $\left(\mathrm{MgSO}_{4}\right)$, filtered, and concentrated. Column chromatography $\left(97: 3, \mathrm{CH}_{2} \mathrm{Cl}_{2}\right.$ :acetone) of the residue yielded $18(434 \mathrm{mg}, 87 \%)$ as a syrup, $[\alpha]_{\mathrm{D}}-13$ ${ }^{\circ}$ (c 1). ${ }^{1} \mathrm{H}$ NMR $\left(\mathrm{CDCl}_{3}\right) \delta 7.40-7.20(\mathrm{~m}, 15 \mathrm{H}, 3 \mathrm{Ph})$, $4.852,4.820,4.600,4.516,4.466$, and 4.403 (6d, each $\left.1 \mathrm{H}, 3 \mathrm{OCH}_{2} \mathrm{Ph}\right), 4.320(\mathrm{~d}, 1 \mathrm{H}, \mathrm{H}-1), 3.962(\mathrm{t}, 1 \mathrm{H}, \mathrm{H}-2)$, 3.937 (d, 1 H, H-4), 3.363 (dd, 1H, H-3), 2.80-2.56 (m, $\left.2 \mathrm{H}, \mathrm{SCH}_{2} \mathrm{CH}_{3}\right), 1.274$ (t, 3H, $\left.\mathrm{SCH}_{2} \mathrm{CH}_{3}\right), 0.887$ (s, 9H, $\left.\mathrm{Si}\left(\mathrm{CH}_{3}\right)_{2} \mathrm{C}\left(\mathrm{CH}_{3}\right)_{3}\right), 0.163$ and 0.023 (2s, each $3 \mathrm{H}$, $\left.\mathrm{Si}\left(\mathrm{CH}_{3}\right)_{2} \mathrm{C}\left(\mathrm{CH}_{3}\right)_{3}\right) ; J_{1,2}=9.2, J_{2,3}=9.0, J_{3,4}=2.7, J_{4,5}$ $=0 \mathrm{~Hz}$.

Ethyl 3,4,6-tri-O-benzyl-2-O-p-methoxybenzyl-1-thio- $\beta$-Dgalactopyranoside (19)

A solution of $17(4.0 \mathrm{~g}, 8.1 \mathrm{mmol})$ and $p$-methoxybenzyl chloride $(1.5 \mathrm{~mL}, 10 \mathrm{mmol})$ in DMF $(9.2 \mathrm{~mL})$ was added dropwise to a suspension of sodium hydride (510 $\mathrm{mg}, 21 \mathrm{mmol})$ in DMF $(7 \mathrm{~mL})$. TLC (7:3, hexane:EtOAc) showed the formation of $19\left(R_{f} 0.59\right)$ to be completed in $90 \mathrm{~min}$. After destroying the excess of sodium hydride with methanol, the mixture was diluted with $\mathrm{CH}_{2} \mathrm{Cl}_{2}$ and washed with water $(2 \times)$, dried $\left(\mathrm{MgSO}_{4}\right)$, filtered, concentrated, and co-concentrated with toluene $(2 \times)$, ethanol $(2 x)$, and $\mathrm{CH}_{2} \mathrm{Cl}_{2}(2 \times)$. Column chromatography $(8: 2$, hexane:EtOAc) of the syrup gave $19(4.6 \mathrm{~g}, 93 \%)$ as a white crystalline solid, mp $64{ }^{\circ} \mathrm{C} ;[\alpha]_{\mathrm{D}}+2^{\circ}$ (c 1). ${ }^{1} \mathrm{H}$ NMR $\left(\mathrm{CDCl}_{3}\right) \delta 7.32-$ $7.27(\mathrm{~m}, 15 \mathrm{H}, 3 \mathrm{Ph}), 6.846\left(\mathrm{~m}, 4 \mathrm{H}, \mathrm{C}_{6} \mathrm{H}_{4} \mathrm{OCH}_{3}\right), 4.943$ and 4.605 (2d, each $\left.1 \mathrm{H}, \mathrm{OCH}_{2} \mathrm{Ph}\right), 4.799$ and 4.713 (2d, each $\left.1 \mathrm{H}, \mathrm{OCH}_{2} \mathrm{Ph}\right), 4.728\left(\mathrm{~s}, 2 \mathrm{H}, \mathrm{OCH}_{2} \mathrm{C}_{6} \mathrm{H}_{4} \mathrm{OCH}_{3}\right)$, 4.454 and $4.396\left(2 \mathrm{~d}\right.$, each $\left.1 \mathrm{H}, \mathrm{OCH}_{2} \mathrm{Ph}\right), 4.408(\mathrm{~d}, 1 \mathrm{H}$, $\mathrm{H}-1), 3.942$ (d, 1H, H-4), 3.805 (t, 1H, H-2), 3.784 (s, $3 \mathrm{H}, \mathrm{C}_{6} \mathrm{H}_{4} \mathrm{OCH}_{3}$ ), 3.544 (dd, $\left.1 \mathrm{H}, \mathrm{H}-3\right), 2.80-2.66(\mathrm{~m}, 2 \mathrm{H}$, $\left.\mathrm{SC} H_{2} \mathrm{CH}_{3}\right), 1.291\left(\mathrm{t}, 3 \mathrm{H}, \mathrm{SCH}_{2} \mathrm{CH}_{3}\right) ; J_{1,2}=9.6, J_{2,3}=$ $9.2, J_{3,4}=2.8, J_{4,5}=0 \mathrm{~Hz}$.

\section{1,2,4,6-Tetra-O-acetyl- $\beta$-D-glucopyranose (2I)}

To a solution of $1,2,4,6$-tetra- $O$-acetyl-3- $O$-benzyl- $\beta$-Dglucopyranose $20^{16}(22.0 \mathrm{~g}, 50.2 \mathrm{mmol})$ in $1: 1$ ethanol:EtOAc $(80 \mathrm{~mL})$ were added $10 \% \mathrm{Pd} / \mathrm{C}(750$ $\mathrm{mg}$ ) and acetic acid ( $1 \mathrm{~mL})$, and the suspension was hydrogenolysed at $4 \mathrm{~kg} / \mathrm{cm}^{2}$ for $24 \mathrm{~h}$. Because of incomplete debenzylation, the hydrogenolysis was repeated twice with intermediate filtration and addition of new catalyst. Then, TLC $(1: 1$, hexane:EtOAc) showed the complete conversion of 20 into $21\left(R_{f} 0.19\right)$. After final filtration, the solution was concentrated, and the residue was crystallised from ethanol to afford 21 $(14.8 \mathrm{~g}, 85 \%)$ as a white crystalline product, $\mathrm{mp} 116$ $121^{\circ} \mathrm{C} ;[\alpha]_{D}+19^{\circ}$ (c 1). ${ }^{1} \mathrm{H}$ NMR $\left(\mathrm{CDCl}_{3}\right) \delta 5.667(\mathrm{~d}$, $1 \mathrm{H}, \mathrm{H}-1$ ), 5.001 (dd, 1H, H-2), 4.981 (t, 1H, H-4), 4.293 (dd, 1H, H-6b), 4.126 (dd, 1H, H-6a), 2.775 (brd, 1H, HO-3), 2.120, 2.111 , and $2.081(3 \mathrm{~s}, 3,6,3 \mathrm{H}, 4 \mathrm{Ac}) ; J_{1,2}=$ $8.3, J_{2,3}=9.6, J_{3,4}=9.7, J_{4,5}=9.7, J_{5,6 \mathrm{a}}=2.2, J_{5,6 \mathrm{~b}}=$ $4.7, J_{6 \mathrm{a}, 6 \mathrm{~b}}=12.4, J_{\mathrm{H}-3, \mathrm{OH}}=6.8 \mathrm{~Hz}$; Anal. calcd for $\mathrm{C}_{14} \mathrm{H}_{20} \mathrm{O}_{10}: \mathrm{C}, 48.28 ; \mathrm{H}, 5.79 \%$; found: $\mathrm{C}, 48.20 ; \mathrm{H}$, $5.78 \%$.

\section{Ethyl 2,4,6-tri-O-acetyl-1-thio- $\beta$-D-glucopyranoside (22)}

To a solution of $21(14.1 \mathrm{~g}, 40.4 \mathrm{mmol})$ in $\mathrm{CH}_{2} \mathrm{Cl}_{2}(120$ $\mathrm{mL}$ ) were added ethanethiol $(3.6 \mathrm{~mL}, 50 \mathrm{mmol})$ and tin(IV) chloride (1.5 mL, $12 \mathrm{mmol}$ ). After stirring for 15 min, TLC (6:4, hexane:EtOAc) showed the disappearance of 21 and the formation of 22 . The mixture was diluted with $\mathrm{CH}_{2} \mathrm{Cl}_{2}$, washed with water, dried $\left(\mathrm{MgSO}_{4}\right)$, filtered, and concentrated. Column chromatography $(6: 4$, hexane:EtOAc) of the residue yielded $22(7.3 \mathrm{~g}, 62 \%)$ as a syrup, $[\alpha]_{D}-25^{\circ}(\mathrm{c} 1) .{ }^{1} \mathrm{H}$ NMR $\left(\mathrm{CDCl}_{3}\right) \delta 4.439$ (d, 1H, H-1), 4.250 (dd, 1H, H6a), 4.157 (dd, 1H, H-6b), 4.735 (brt, 1H, H-3), 3.643 (ddd, $1 \mathrm{H}, \mathrm{H}-5$ ), 2.80-2.60 (m, $\left.2 \mathrm{H}, \mathrm{SCH}_{2} \mathrm{CH}_{3}\right), 2.137$, 2.166 , and 2.078 ( $3 \mathrm{~s}$, each $3 \mathrm{H}, 3 \mathrm{Ac}), 1.275$ (t, $3 \mathrm{H}$, $\left.\mathrm{SCH}_{2} \mathrm{CH}_{3}\right) ; J_{1,2}=10.0, J_{5,6 \mathrm{a}}=2.5, J_{5,6 \mathrm{~b}}=5.1, J_{6 \mathrm{a}, 6 \mathrm{~b}}=$ $12.3 \mathrm{~Hz}$; Anal. calcd for $\mathrm{C}_{14} \mathrm{H}_{22} \mathrm{O}_{8} \mathrm{~S}$ : C, $47.99 ; \mathrm{H}, 6.33$ $\%$; found: C, $47.84 ; \mathrm{H}, 6.38 \%$.

Ethyl 2,4,6-tri- O- acetyl-3-0- (3,4,6-tri-O-benzyl-2-0-1 butyldimethylsilyl- $\alpha$-D-galactopyranosyl)-l-thio- $\beta-D$ gluco-pyranoside (23)

A mixture of $18(102 \mathrm{mg}, 0.18 \mathrm{mmol}), 22(57 \mathrm{mg}, 0.16$ $\mathrm{mmol}$ ) and $4 \AA$ molecular sieves (200 mg) in 1:5 1,2dichloroethane:diethyl ether $(6 \mathrm{~mL})$ was stirred for 30 min at $-60{ }^{\circ} \mathrm{C}$, then iodonium dicollidineperchlorate $(0.19 \mathrm{~g}, 0.41 \mathrm{mmol})$ was added. After $30 \mathrm{~min}$, TLC (6:4, 
hexane:EtOAc) showed the disappearance of 18 and the appearance of a new spot $\left(R_{f} 0.54\right)$. The mixture was diluted with $\mathrm{CH}_{2} \mathrm{Cl}_{2}$, filtered over Celite, washed with aq. $10 \%$ sodium thiosulfate and water $(2 \times)$, dried ( $\mathrm{MgSO}_{4}$ ), filtered, and concentrated. Column chromatography (Sephadex LH-20 in $1: 1 \mathrm{CH}_{2} \mathrm{Cl}_{2}$ : methanol, then silica using 6:4, hexane:EtOAc) of the residue afforded $23(70 \mathrm{mg}, 49 \%)$ as a syrup. ${ }^{1} \mathrm{H}$ NMR $\left(\mathrm{CDCl}_{3}\right) \delta$ 7.33-7.24 (m, 15H, $\left.3 \mathrm{Ph}\right), 4.850$ (d, 1H, H$\left.1^{\prime}\right), 4.803$ and 4.465 (2d, each $\left.1 \mathrm{H}, \mathrm{OCH}_{2} \mathrm{Ph}\right), 4.612(2 \mathrm{~d}$, each $\left.1 \mathrm{H}, \mathrm{OCH}_{2} \mathrm{Ph}\right), 4.526$ and 4.397 (2d, each $1 \mathrm{H}$, $\mathrm{OCH}_{2} \mathrm{Ph}$ ), 4.346 (d, 1H, H-1), 4.140 (dd, 1H, H-2'), 3.911 (dd, 1H, H-4'), 3.749 (t, 1H, H-3), 2.77-2.64 (m, $\left.2 \mathrm{H}, \mathrm{SCH}_{2} \mathrm{CH}_{3}\right), 2.091$ and $2.013(2 \mathrm{~s}, 6,3 \mathrm{H}, 3 \mathrm{Ac}), 1.257$ (t, 3H, $\left.\mathrm{SCH}_{2} \mathrm{CH}_{3}\right), 0.899$ (s, 9H, $\left.\mathrm{Si}\left(\mathrm{CH}_{3}\right)_{2} \mathrm{C}\left(\mathrm{CH}_{3}\right)_{3}\right)$, 0.141 and $0.013\left(2 \mathrm{~s}\right.$, each $\left.3 \mathrm{H}, \mathrm{Si}\left(\mathrm{CH}_{3}\right)_{2} \mathrm{C}\left(\mathrm{CH}_{3}\right)_{3}\right) ; J_{1,2}=$ $10.0, J_{1^{\prime}, 2^{\prime}}=3.2, J_{2^{\prime}, 3^{\prime}}=10.3, J_{3^{\prime}, 4^{\prime}}=2.6, J_{4^{\prime}, 5^{\prime}}=1.3 \mathrm{~Hz}$.

Ethyl 2,4,6-tri-O-acetyl-3-0-(3,4,6-tri-O-benzyl-2-O-pmethoxybenzyl- $\alpha$ - D-galactopyranosyl)- 1 - thio- $\beta$-D-glucopyranoside (24)

A mixture of $19(2.41 \mathrm{~g}, 3.92 \mathrm{mmol}), 22(1.25 \mathrm{~g}, 3.57$ $\mathrm{mmol}$ ) and $4 \AA$ molecular sieves $(5 \mathrm{~g})$ in $1: 51,2-$ dichloroethane:diethyl ether $(6 \mathrm{~mL})$ was stirred for 15 min at room temperature, then iodonium dicollidineperchlorate $(1.84 \mathrm{~g}, 3.97 \mathrm{mmol})$ was added. After $30 \mathrm{~min}$, TLC (6:4, hexane:EtOAc) showed the disappearance of the reactants and the formation of 24 $\left(R_{f} 0.54\right)$. The mixture was diluted with $\mathrm{CH}_{2} \mathrm{Cl}_{2}$, filtered over Celite, washed with aq. $10 \%$ sodium thiosulfate and water $(2 \times)$, dried $\left(\mathrm{MgSO}_{4}\right)$, filtered, and concentrated. Column chromatography $(6: 4$, hexane: EtOAc) of the residue afforded $24(2.68 \mathrm{~g}, 83 \%)$ as a syrup, $[\alpha]_{\mathrm{D}}+15^{\circ}$ (c 1). ${ }^{1} \mathrm{H}$ NMR $\left(\mathrm{CDCl}_{3}\right) \delta 7.50-6.75$ $\left(\mathrm{m}, 19 \mathrm{H}, 3 \mathrm{Ph}\right.$ and $\left.\mathrm{C}_{6} \mathrm{H}_{4} \mathrm{OCH}_{3}\right), 4.837$ (d, 1H, H-1'), $4.886,4.729,4.655,4.636,4.557,4.519,4.505$, and 4.382 (8d, each $1 \mathrm{H}, 3 \mathrm{OCH}_{2} \mathrm{Ph}$ and $\mathrm{OCH}_{2} \mathrm{C}_{6} \mathrm{H}_{4} \mathrm{OCH}_{3}$ ), 4.324 (d, 1H, H-1), 4.231 (dd, 1H, H-6b), 4.147 (dd, 1H, H6a), 4.020 (m, 1H, H-5'), 3.955 (dd, 1H, H-4'), 3.913 (dd, 1H, H-2'), 3.802 (dd, 1H, H-3'), 3.704 (t, 1H, H-3), 2.80-2.60 (m, 2H, SCH $\left.\mathrm{CH}_{3}\right), 2.079,1.997$, and 1.806 (3s, each $3 \mathrm{H}, 3 \mathrm{Ac}), 1.255\left(\mathrm{t}, 3 \mathrm{H}, \mathrm{SCH}_{2} \mathrm{CH}_{3}\right) ; J_{1,2}=$ $10.0, J_{5,6 \mathrm{a}}=2.4, J_{5,6 \mathrm{~b}}=4.8, J_{6 \mathrm{a}, 6 \mathrm{~b}}=12.2, J_{1^{\prime}, 2^{\prime}}=3.2$, $J_{2^{\prime}, 3^{\prime}}=10.2, J_{3^{\prime}, 4^{\prime}}=2.7, J_{4^{\prime}, 5^{\prime}}=1.5 \mathrm{~Hz}$; Anal, calcd for $\mathrm{C}_{49} \mathrm{H}_{58} \mathrm{O}_{14} \mathrm{~S}: \mathrm{C}, 65.17 ; \mathrm{H}, 6.47 \%$; found: $\mathrm{C}, 65.02 ; \mathrm{H}$, $6.43 \%$.

Ethyl 2,4,6-tri-O-benzyl-3-0-(3,4,6-tri-O-benzyl-2-0-pmethoxybenzyl- $\alpha$-D-galactopyranosyl)- 1 -thio- $\beta-D-$ - luc 0 pyranoside (26)

To a solution of $24(400 \mathrm{mg}, 0.44 \mathrm{mmol})$ in methanol (10 mL) was added sodium methoxide ( $\mathrm{pH} \mathrm{10).} \mathrm{After}$ refluxing overnight, TLC (95:5, $\mathrm{CH}_{2} \mathrm{Cl}_{2}$ :acetone) showed the formation of $25\left(R_{f} 0.05\right)$. The mixture was neutralised using Dowex-50 $\left(\mathrm{H}^{+}\right)$resin, filtered, and concentrated to give 25 in a quantitative yield. A solution of 25 (300 $\mathrm{mg}, 0.41 \mathrm{mmol})$ and benzyl bromide (0.16 mL, $1.35 \mathrm{mmol}$ ) in DMF (4 mL) was added dropwise to a suspension of sodium hydride $(0.11 \mathrm{~g}, 4.6$ mmol) in DMF $(2 \mathrm{~mL})$. TLC $(7: 3$, hexane:EtOAc) showed that the benzylation was completed in $90 \mathrm{~min}$ $\left(26, R_{f}\right.$ 0.47). After destroying the excess of sodium hydride with methanol, the mixture was diluted with $\mathrm{CH}_{2} \mathrm{Cl}_{2}$, washed with water $(2 \times)$, dried $\left(\mathrm{MgSO}_{4}\right)$, filtered, and concentrated. Column chromatography (8:2, hexane:EtOAc) of the residue gave $26(383 \mathrm{mg}, 83$ \%) as a syrup, $[\alpha]_{\mathrm{D}}+26^{\circ}$ (c 1). ${ }^{1} \mathrm{H}$ NMR $\left(\mathrm{CDCl}_{3}\right) \delta$ $7.40-6.65\left(\mathrm{~m}, 34 \mathrm{H}, 6 \mathrm{Ph}\right.$ and $\left.\mathrm{C}_{6} \mathrm{H}_{4} \mathrm{OCH}_{3}\right), 5.580(\mathrm{~d}, 1 \mathrm{H}$, $\left.\mathrm{H}-\mathrm{l}^{\prime}\right), 4.968,4.863,4.853,4.766,4.698,4.673,4.631$, $4.620,4.505,4.494(10 \mathrm{~d}$, each $1 \mathrm{H}), 4.538(\mathrm{~d}, 2 \mathrm{H})$, and $4.203(\mathrm{~s}, 2 \mathrm{H})\left(6 \mathrm{OCH}_{2} \mathrm{Ph}\right.$ and $\left.\mathrm{OCH}_{2} \mathrm{C}_{6} \mathrm{H}_{4} \mathrm{OCH}_{3}\right), 4.380$ (d, 1H, H-1), 4.047 (dd, 1H, H-2'), 4.000 (t, 1H, H-2), 3.969 (dd, $1 \mathrm{H}, \mathrm{H}-3$ '), 3.764 (s, 3H, $\mathrm{C}_{6} \mathrm{H}_{4} \mathrm{OCH}_{3}$ ), 2.84 $2.62\left(\mathrm{~m}, 2 \mathrm{H}, \mathrm{SCH}_{2} \mathrm{CH}_{3}\right), 1.298\left(\mathrm{t}, 3 \mathrm{H}, \mathrm{SCH}_{2} \mathrm{CH}_{3}\right) ; J_{1,2}$ $=9.6, J_{2,3}=8.9, J_{1^{\prime}, 2^{\prime}}=3.3, J_{2^{\prime}, 3^{\prime}}=10.2, J_{3^{\prime}, 4^{\prime}}=2.6 \mathrm{~Hz}$.

O-(3,4,6-Tri-O-benzyl-2-O-p-methoxybenzyl- $\alpha$-D-galacto-

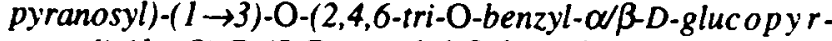
anosyl $)-(1 \rightarrow 3)-0-(2-0$-acetyl-4-0-benzyl- $\alpha$-L-rhamnopyranosyl)- $(1 \rightarrow 4)$ - 5-0-allyl-1-0-benzoyl-2,3-di-O-benzylD-ribitol $(31 \alpha \beta)$

A mixture of 26 (121 $\mathrm{mg}, 0.12 \mathrm{mmol}), 11$ (78 $\mathrm{mg}, 0.095$ mmol) and $4 \AA$ molecular sieves $(0.3 \mathrm{~g})$ in $1: 51,2-$ dichloroethane:diethyl ether $(3 \mathrm{~mL})$ was stirred for 30 min, after which iodonium dicollidineperchlorate (107 $\mathrm{mg}, 0.23 \mathrm{mmol})$ was added. TLC $(8: 2$, hexane:EtOAc) showed the disappearance of the reactants and the formation of $31\left(R_{f} 0.25\right)$ in $90 \mathrm{~min}$. Then, the mixture was diluted with $\mathrm{CH}_{2} \mathrm{Cl}_{2}$, filtered, and washed with aq. $10 \%$ sodium thiosulfate and water, dried $\left(\mathrm{MgSO}_{4}\right)$, filtered, and concentrated. Column chromatography (Sephadex $\mathrm{LH}-20$ in $1: 1 \mathrm{CH}_{2} \mathrm{Cl}_{2}$ :methanol, then silica using 4:1 hexane: EtOAc) of the residue afforded $31 \alpha \beta$ (115 mg, $67 \%, \alpha: \beta$ ratio $1: 1$ ) as a syrup. ' $H$ NMR $\left(\mathrm{CDCl}_{3}\right) \delta 7.95-6.50\left(\mathrm{~m}, 54 \mathrm{H}, 10 \mathrm{Ph}\right.$ and $\left.\mathrm{C}_{6} \mathrm{H}_{4} \mathrm{OCH}_{3}\right)$, $5.858\left(\mathrm{~m}, 1 \mathrm{H}, \mathrm{OCH}_{2} \mathrm{CH}=\mathrm{CH}_{2}\right), 5.591$ (d, $1 \mathrm{H}, \mathrm{H}-1^{\prime \prime \prime}$, $31 \alpha$ ), 5.504 (d, 1H, H-1"', $31 \beta$ ), 5.310 (d, 1H, H-l", $31 \alpha), 5.414(\mathrm{~d}, 1 \mathrm{H}, \mathrm{H}-1,31 \beta), 5.116\left(\mathrm{~d}, 1 \mathrm{H}, \mathrm{H}-\mathrm{l}^{\prime}, 31 \alpha\right)$, 3.671 (s, 3H, $\left.\mathrm{C}_{6} \mathrm{H}_{4} \mathrm{OCH} 3,31 \beta\right), 3.664$ (s, 3H, $\mathrm{C}_{6} \mathrm{H}_{4} \mathrm{OCH}_{3}, 31 \alpha$ ), 2.125 (s, 3H, Ac, 31ß), 1.857 (s, 3H, Ac, $31 \alpha$ ), 1.192 (d, 3H, 3 H-6, $31 \alpha$ ), 1.116 (d, 3H, 3 H-

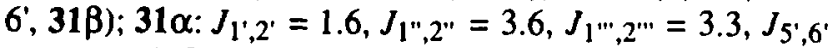
$=6.1 \mathrm{~Hz} ; 31 \beta: J_{1^{\prime}, 2^{\prime}}=1.7, J_{1^{\prime \prime}, 2^{\prime \prime \prime}}=3.5, J_{5^{\prime}, 6^{\prime}}=6.1 \mathrm{~Hz}$.

Ethyl 4,6-0-benzylidene-3-O-(3,4,6-tri-O-benzyl-2-0-pmethoxybenzyl- $\alpha-D$-galactopyranosyl)-1-thio- $\beta-D-g l$ uco pyranoside (27)

Compound 24 (3.28 g, $3.63 \mathrm{mmol})$ was converted into 25 as described above. Crude 25 was dissolved in DMF (10 mL) and $\alpha, \alpha$-dimethoxytoluene $(0.65 \mathrm{~mL})$, and $p$ toluenesulfonic acid ( $\mathrm{pH} \mathrm{2)}$ was added. The mixture was stirred for $5 \mathrm{~h}$ at $50^{\circ} \mathrm{C}$, when TLC $\left(95: 5, \mathrm{CH}_{2} \mathrm{Cl}_{2}\right.$ : acetone) showed the formation of $27\left(R_{f} 0.56\right)$. Solid $\mathrm{NaHCO}_{3}$ was added, and the mixture was diluted with EtOAc, washed with aq. $10 \% \mathrm{NaHCO}_{3}$ and water $(3 \times)$, dried $\left(\mathrm{MgSO}_{4}\right)$, filtered, and concentrated. Column chromatography $(6: 4$, hexane:EtOAc) of the residue afforded $27(1.87 \mathrm{~g}, 60 \%$ from 24$)$ as a syrup, $[\alpha]_{D}+8^{\circ}$ 
(c 1). ${ }^{1} \mathrm{H}$ NMR $\left(\mathrm{CDCl}_{3}\right) \delta 7.45-7.25(\mathrm{~m}, 20 \mathrm{H}, 4 \mathrm{Ph})$, $7.00-6.55\left(\mathrm{~m}, 4 \mathrm{H}, \mathrm{C}_{6} \mathrm{H}_{4} \mathrm{OCH}_{3}\right), 5.498(\mathrm{~s}, 1 \mathrm{H}, \mathrm{CHPh})$, $5.422(\mathrm{~d}, 1 \mathrm{H}, \mathrm{H}-1), 4.280(\mathrm{~d}, 1 \mathrm{H}, \mathrm{H}-1), 3.734(\mathrm{~s}, 3 \mathrm{H}$, $\left.\mathrm{C}_{6} \mathrm{H}_{4} \mathrm{OCH}_{3}\right), 2.76-2.63\left(\mathrm{~m}, 2 \mathrm{H}, \mathrm{SCH}_{2} \mathrm{CH}_{3}\right), 1.292(\mathrm{t}$, $\left.3 \mathrm{H}, \mathrm{SCH}_{2} \mathrm{CH}_{3}\right) ; J_{1,2}=9.7, J_{1^{\prime}, 2^{\prime}}=3.5 \mathrm{~Hz}$.

Ethyl 2-0-benzyl-4,6-0-benzylidene-3-0-(3,4,6-tri-0benzyl-2-O-p-methoxybenzyl- $\alpha$-D-galactopyranosyl)-Ithio- $\beta$-D-glucopyranoside (28)

A solution of $27(330 \mathrm{mg}, 0.38 \mathrm{mmol})$ and benzyl bromide $(54 \mu \mathrm{L}, 0.45 \mathrm{mmol})$ in DMF ( $3 \mathrm{~mL})$ was added dropwise to sodium hydride $(30 \mathrm{mg}, 1.3 \mathrm{mmol})$. TLC (98:2, $\mathrm{CH}_{2} \mathrm{Cl}_{2}$ :acetone, $R_{f} 0.30$ ) showed the benzylation to be completed in $15 \mathrm{~min}$. After destroying the excess of sodium hydride with methanol, the mixture was diluted with EtOAc, washed with water $(3 \times)$, dried $\left(\mathrm{MgSO}_{4}\right)$, filtered, and concentrated. Column chromatography $\left(98: 2, \mathrm{CH}_{2} \mathrm{Cl}_{2}\right.$ :acetone) of the residue gave $28(322 \mathrm{mg}, 89 \%)$ as a syrup, $[\alpha]_{D}+24^{\circ}(\mathrm{c} 1){ }^{1} \mathrm{H}$ NMR $\left(\mathrm{CDCl}_{3}\right) \delta 7.35-7.20(\mathrm{~m}, 25 \mathrm{H}, 5 \mathrm{Ph}), 7.00-6.60$ (m, $\left.4 \mathrm{H}, \mathrm{C}_{6} H_{4} \mathrm{OCH}_{3}\right), 5.668\left(\mathrm{~d}, 1 \mathrm{H}, \mathrm{H}-\mathrm{l}^{\prime}\right), 5.469(\mathrm{~s}, 1 \mathrm{H}$, $\mathrm{CHPh}), 4.940,4.856,4.715,4.602,4.573,4.478$, and $4.456\left(7 \mathrm{~d}, 1,2,1,1,1,1,1 \mathrm{H}, 4 \mathrm{OCH}_{2} \mathrm{Ph}\right), 4.501(\mathrm{~d}, 1 \mathrm{H}, \mathrm{H}-$ 1), 3.963 (dd, 1H, H-2'), 3.891 (dd, 1H, H-3'), 3.727 (s, $\left.\left.3 \mathrm{H}, \mathrm{C}_{6} \mathrm{H}_{4} \mathrm{OCH}\right)_{3}\right), 3.443$ (dt, $\left.1 \mathrm{H}, \mathrm{H}-5\right), 3.417$ (dd, $1 \mathrm{H}, \mathrm{H}-$ 6a), 3.260 (dd, 1H, H-6b), 2.84-2.66 (m, 2H, $\left.\mathrm{SCH}_{2} \mathrm{CH}_{3}\right), 1.312\left(\mathrm{t}, 3 \mathrm{H}, \mathrm{SCH}_{2} \mathrm{CH}_{3}\right) ; J_{1,2}=9.7, J_{4,5}=$ $10.5, J_{5,6 \mathrm{a}}=6.5, J_{5,6 \mathrm{~b}}=7.0, J_{6 \mathrm{a}, 6 \mathrm{~b}}=9.4, J_{1^{\prime}, 2^{\prime}}=3.4, J_{2^{\prime}, 3^{\prime}}$ $=10.2, J_{3 ; 4^{\circ}}=2.6 \mathrm{~Hz}$; Anal. calcd for $\mathrm{C}_{57} \mathrm{H}_{62} \mathrm{O}_{11} \mathrm{~S}: \mathrm{C}$, 71.68; H, $6.54 \%$; found: $\mathrm{C}, 71.48 ; \mathrm{H}, 6.48 \%$.

Ethyl 2-O-benzyl-3-O-(3,4,6-tri-O-benzyl-2-O-p-methoxybenzyl- $\alpha$-D-galactopyranosyl)-1-thio- $\beta$-D-glucopyranoside (29)

A solution of $28(51 \mathrm{mg}, 53 \mu \mathrm{mol})$ in $\mathrm{CH}_{2} \mathrm{Cl}_{2}(2 \mathrm{~mL})$ was mixed with $1: 2$ water:trifluoroacetic acid $(6 \mu \mathrm{L})$ under vigorous stirring. After $45 \mathrm{~h}$, TLC $6: 4$, hexane:EtOAc) showed the disappearance of $28\left(R_{f}\right.$ $0.59)$ and the appearance of two new spots $\left(R_{f} 0.19\right.$ and $R_{f} 0.09$ ). Solid $\mathrm{NaHCO}_{3}$ was added, and the mixture was diluted with $\mathrm{CH}_{2} \mathrm{Cl}_{2}$, washed with water $(3 \times)$, dried $\left(\mathrm{MgSO}_{4}\right)$, filtered, and concentrated. Column chromatography (6:4, hexane: EtOAc) of the residue afforded $29\left(R_{f} 0.19,19 \mathrm{mg}, 41 \%\right)$ as a syrup, $[\alpha]_{\mathrm{D}}+9^{\circ}$ (c 0.25 ), and part of the lower moving component $\left(R_{f}\right.$ $0.09,4 \mathrm{mg}, 9 \%) .{ }^{1} \mathrm{H}$ NMR $\left(\mathrm{CDCl}_{3}\right) \delta$ 7.45-6.70 (m, $24 \mathrm{H}, 4 \mathrm{Ph}$ and $\mathrm{C}_{6} \mathrm{H}_{4} \mathrm{OCH}_{3}$ ), 5.022 (d, $\left.1 \mathrm{H}, \mathrm{H}-\mathrm{l}^{\prime}\right), 4.899$, $4.805,4.783,4.705,4.665,4.543,4.254$, and 4.199 (8d, each $1 \mathrm{H}, 3 \mathrm{OCH}_{2} \mathrm{Ph}$ and $\left.\mathrm{OCH}_{2} \mathrm{C}_{6} \mathrm{H}_{4} \mathrm{OCH}_{3}\right), 4.758(\mathrm{~s}$, $2 \mathrm{H}, \mathrm{OCH}_{2} \mathrm{Ph}$ ), 4.443 (d, $1 \mathrm{H}, \mathrm{H}-1$ ), 4.155 (brdd, $1 \mathrm{H}, \mathrm{H}-$ 4), 4.082 (dd, 1H, H-2'), 4.000 (dd, 1H, H-3'), 3.775 (s, $\left.3 \mathrm{H}, \mathrm{C}_{6} \mathrm{H}_{4} \mathrm{OCH}_{3}\right), 2.80-2.76\left(\mathrm{~m}, 2 \mathrm{H}, \mathrm{SCH}_{2} \mathrm{CH}_{3}\right), 1.299$ (t. $\left.3 \mathrm{H}, \mathrm{SCH}_{2} \mathrm{CH}_{3}\right) ; J_{1,2}=9.8, J_{1^{\prime}, 2^{\prime}}=3.3, J_{2^{\prime}, 3^{\prime}}=9.8$, $J_{3^{\prime}, 4^{\prime}}=2.6 \mathrm{~Hz}$; Anal. calcd for $\mathrm{C}_{50} \mathrm{H}_{58} \mathrm{O}_{11} \mathrm{~S}: \mathrm{C}, 69.26 ; \mathrm{H}$, $6.74 \%$; found: C, $69.38 ; \mathrm{H}, 6.77 \%$.

Ethyl 4,6-di-O-acetyl-2-O-benzyl-3-O-(3,4,6-tri-O-benzyl2-O-p- methoxybenzyl- $\alpha$ - $D$ - galactopyranosyl)-1-thio- $\beta$ D-glucopyranoside (30)

A solution of $29(40 \mathrm{mg}, 46 \mu \mathrm{mol})$ in $2: 1$ pyridine:acetic anhydride $(3 \mathrm{~mL})$ was stirred for $24 \mathrm{~h}$, when TLC $(6: 4$, hexane:EtOAc) showed the formation of $\mathbf{3 0}$. The mixture was co-concentrated with toluene $(3 \times)$, ethanol $(3 x)$, and $\mathrm{CH}_{2} \mathrm{Cl}_{2}(3 \times)$. Column chromatography $(7: 3$, hexane: EtOAc) of the residue gave $30(32 \mathrm{mg}, 73 \%)$ as a white solid, mp $104{ }^{\circ} \mathrm{C} ;[\alpha]_{\mathrm{D}}+29^{\circ}$ (c 1). ${ }^{1} \mathrm{H}$ NMR $\left(\mathrm{CDCl}_{3}\right) \delta 7.45-6.80\left(\mathrm{~m}, 24 \mathrm{H}, 4 \mathrm{Ph}\right.$ and $\left.\mathrm{C}_{6} \mathrm{H}_{4} \mathrm{OCH}_{3}\right)$, $5.211\left(\mathrm{~d}, 1 \mathrm{H}, \mathrm{H}-1^{\prime}\right), 5.142(\mathrm{t}, 1 \mathrm{H}, \mathrm{H}-4), 4.897,4.845$, $4.788,4.763,4.674$, and $4.518(6 \mathrm{~d}$, each $1 \mathrm{H}, 3$ $\mathrm{OCH}_{2} \mathrm{Ph}$ ), 4.605 (s, $\left.2 \mathrm{H}, \mathrm{OCH}_{2} \mathrm{Ph}\right), 4.392$ (d, $1 \mathrm{H}, \mathrm{H}-1$ ), 4.284 (s, $\left.2 \mathrm{H}, \mathrm{OCH}_{2} \mathrm{C}_{6} \mathrm{H}_{4} \mathrm{OCH}_{3}\right), 4.198$ (dd, $1 \mathrm{H}, \mathrm{H}-6 \mathrm{~b}$ ), 4.069 (dd, 1H, H-6a), 3.779 (s, $3 \mathrm{H}, \mathrm{C}_{6} \mathrm{H}_{4} \mathrm{OCH}_{3}$ ), 3.737 (dd, $1 \mathrm{H}, \mathrm{H}-4$ ), 2.85-2.65 (m, $2 \mathrm{H}, \mathrm{SCH}_{2} \mathrm{CH}_{3}$ ), 2.073 and 1.825 (2s, each $3 \mathrm{H}, 2 \mathrm{Ac}$ ), $1.320\left(\mathrm{t}, 3 \mathrm{H}, \mathrm{SCH}_{2} \mathrm{CH}_{3}\right) ; J_{1,2}$ $=9.8, J_{3,4}=9.5, J_{4.5}=9.5, J_{5,6 \mathrm{a}}=2.4, J_{5,6 \mathrm{~b}}=5.2, J_{6 \mathrm{a}, 6 \mathrm{~b}}$ $=12.2, J_{1^{\prime}, 2^{\prime}}=3.3, J_{3^{\prime}, 4^{\prime}}=1.2, J_{4^{\prime}, 5^{\prime}}=2.6 \mathrm{~Hz}$; Anal. calcd for $\mathrm{C}_{54} \mathrm{H}_{62} \mathrm{O}_{13} \mathrm{~S}$ : C, $68.19 ; \mathrm{H}, 6.57 \%$; found: $\mathrm{C}, 68.40$; $\mathrm{H}, 6.50 \%$.

O-(3,4,6-Tri-O-benzyl-2-O-p-methoxybenzyl- $\alpha$-D-galactopyranosyl)-( $(1 \rightarrow 3)-0-(2,4,6-t r i-0-$ benzyl- $\alpha-D-g$ lucopyr-

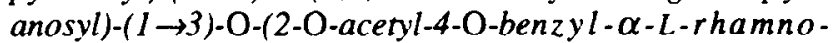
pyranosyl)-(l-4)-1-O-benzoyl-2,3-di-O-benzyl-D-ribitol (33)

To a solution of $31 \alpha \beta(105 \mathrm{mg}, 0.057 \mathrm{mmol})$ and diazabicyclo[2.2.2] octane $(38 \mathrm{mg}, 0.34 \mathrm{mmol})$ in $8: 3: 1$ ethanol:toluene:water $(9 \mathrm{~mL})$ was added tris(triphenylphosphine)rhodium(I) chloride $(20 \mathrm{mg})$. After refluxing for $90 \mathrm{~min}$, TLC $(3: 1$, hexane:EtOAc) showed a complete conversion of the allyl into the propenyl function $\left(R_{f} 0.43\right)$. The mixture was concentrated, dissolved in acetone $(4 \mathrm{~mL})$, and to the solution were added mercuric oxide $(30 \mathrm{mg}, 0.14 \mathrm{mmol})$ and mercuric chloride ( $26 \mathrm{mg}, 0.11 \mathrm{mmol}$ ). After stirring the mixture for $30 \mathrm{~min}$, TLC $(8: 2$, hexane:EtOAc) showed the depropenylation to be completed. Then, the mixture was diluted with $\mathrm{CH}_{2} \mathrm{Cl}_{2}$, filtered, washed with aq. $5 \% \mathrm{KI}$ and water, dried $\left(\mathrm{MgSO}_{4}\right)$, filtered, and concentrated. Preparative TLC (8:2 hexane: EtOAc) made it possible to separate the $\alpha / \beta$ coupling mixture, yielding 33 (36 $\mathrm{mg}, 37 \%)$ as a syrup. ${ }^{1} \mathrm{H}$ NMR $\left(\mathrm{CDCl}_{3}\right) \delta 7.95-6.50$ $\left(\mathrm{m}, 54 \mathrm{H}, 10 \mathrm{Ph}\right.$ and $\left.\mathrm{C}_{6} \mathrm{H}_{4} \mathrm{OCH}_{3}\right), 5.579\left(\mathrm{~d}, 1 \mathrm{H}, \mathrm{H}-1^{\prime \prime \prime}\right)$, 5.289 (d, 1H, H-1"), 5.005 (d, 1H, H-1'), 3.668 (s, 3H, $\mathrm{C}_{6} \mathrm{H}_{4} \mathrm{OCH}_{3}$ ), 1.858 (s, 3H, Ac), 1.220 (d, 3H, $\left.3 \mathrm{H}-6^{\prime}\right)$; $J_{1^{\prime}, 2^{\prime}}=1.8, J_{1^{\prime \prime}, 2^{\prime \prime}}=3.5, J_{1^{\prime \prime \prime}, 2^{\prime \prime \prime}}=3.4, J_{5^{\prime}, 6^{\prime}}=6.2 \mathrm{~Hz}$; Anal. calcd for $\mathrm{C}_{103} \mathrm{H}_{110} \mathrm{O}_{22}: \mathrm{C}, 72.77 ; \mathrm{H}, 6.52 \%$; found: $\mathrm{C}$, $72.61 ; \mathrm{H}, 6.45 \%$.

O-(3,4,6-Tri-O-benzyl-2-O-p-methoxybenzyl- $\alpha$-D-galacto-

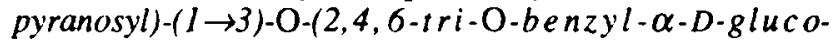
pyranosyl)-( $(1 \rightarrow 3)-\mathrm{O}-(2-\mathrm{O}-$ aceryl-4-O-benzyl- $\alpha$-L-rhamnopyrano-syl)-( $1 \rightarrow 4)-1$-O-benzoyl-2,3-di-O-benzyl-5-0-(3-N[benzyl-oxycarbonyl]-aminopropyl phosphate)-D-ribitol (35)

A mixture of $33(29 \mathrm{mg}, 16 \mu \mathrm{mol})$ and $3-N-$ (benzyloxycarbonyl)-aminopropyl phosphonate $(20 \mathrm{mg}$, $78 \mu \mathrm{mol}$; prepared by phosphonylation of $3-\mathrm{N}$ (benzyloxycarbonyl)-aminopropanol with 2-chloro- $4 \mathrm{H}$ 1,3,2-benzodioxaphosphorin-2-one ${ }^{14}$ ), were co- 
concentrated with pyridine $(2 \times)$. Then, pyridine $(0.5$ $\mathrm{mL}$ ) was added, and $0.5 \mathrm{~mL}$ of a stock solution of pivaloyl chloride $(0.18 \mathrm{~mL})$ in pyridine $(10 \mathrm{~mL})$ was injected into the solution. After $20 \mathrm{~min}$, TLC (9:1, $\mathrm{CH}_{2} \mathrm{Cl}_{2}$ :acetone) showed the formation of $34\left(R_{f} 0.45\right)$. The mixture was diluted with $\mathrm{CH}_{2} \mathrm{Cl}_{2}$, washed with saline and water, dried $\left(\mathrm{Na}_{2} \mathrm{SO}_{4}\right)$, filtered, and concentrated. Column chromatography (9:1, $\mathrm{CH}_{2} \mathrm{Cl}_{2}$ :acetone) of the residue afforded $34(16 \mathrm{mg}, 53$ $\%)$.

A $0.2 \mathrm{M}$ solution of $\mathrm{I}_{2}$ in $95: 5$ pyridine:water $(2 \mathrm{~mL})$ was added to 34 (16 mg. $8.5 \mu \mathrm{mol}$ ). After $20 \mathrm{~min}$, TLC ( $9: 1$, $\mathrm{CH}_{2} \mathrm{Cl}_{2}$ :acetone) showed the disappearance of 34 and a new spot on the baseline. The mixture was diluted with $\mathrm{CH}_{2} \mathrm{Cl}_{2}$, washed with aq. $5 \%$ sodium thiosulfate, aq. 10 $\% \mathrm{NaHCO}_{3}$, and water, dried $\left(\mathrm{Na}_{2} \mathrm{SO}_{4}\right)$, filtered, and concentrated. Column chromatography $\left(9: 1 \mathrm{CH}_{2} \mathrm{Cl}_{2}\right.$ : acetone, then 9:1 $\mathrm{CH}_{2} \mathrm{Cl}_{2}$ :methanol) of the residue gave 35 (11 mg, $65 \%)$. ${ }^{1} \mathrm{H}$ NMR $\left(\mathrm{CDCl}_{3}\right) \delta 7.90-6.45(\mathrm{~m}$, $54 \mathrm{H}, 10 \mathrm{Ph}$ and $\left.\mathrm{C}_{6} \mathrm{H}_{4} \mathrm{OCH}_{3}\right), 5.575$ (d, $\left.1 \mathrm{H}, \mathrm{H}-\mathrm{l}^{\prime \prime}\right), 5.433$ (d, 1H, H-2'), 5.253 (d, 1H, H-1"), 5.050 (d, 1H, H-1'), 3.666 (s, $\left.3 \mathrm{H}, \quad \mathrm{C}_{6} \mathrm{H}_{4} \mathrm{OCH}_{3}\right), 3.424(\mathrm{~m}, 2 \mathrm{H}$, $\left.\mathrm{O}\left(\mathrm{CH}_{2}\right)_{2} \mathrm{CH}_{2} \mathrm{NH}\right), 3.12\left(\mathrm{~m}, 2 \mathrm{H}, \mathrm{OCH}_{2}\left(\mathrm{CH}_{2}\right)_{2} \mathrm{NH}\right), 1.756$

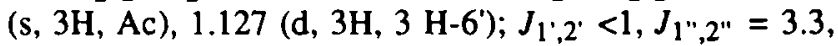
$J_{1}^{\prime \prime \prime}, 2^{\prime \prime}=3.3, J_{5^{\prime}, 6^{\prime}}=5.7 \mathrm{~Hz}$; Anal. calcd for $\mathrm{C}_{114} \mathrm{H}_{124} \mathrm{NO}_{27} \mathrm{P}$ : $\mathrm{C}, 69.46 ; \mathrm{H}, 6.34 \%$; found: $\mathrm{C}, 69.31$; $\mathrm{H}, 6.44 \%$.

O- $\alpha$-D-Galactopyranosyl-( $(I \rightarrow 3)-0-\alpha$-D-glucopyranosyl$(1 \rightarrow 3)-0-\alpha-L-r h a m n o p y r a n o s y l-(1 \rightarrow 4)-(5-0-[3-a m i n o-$ propyl phosphate])-D-ribitol (1)

A solution of $35(7.3 \mathrm{mg}, 2.7 \mu \mathrm{mol})$ in $2: 1$ methanol:aq. $25 \%$ ammonia $(1.5 \mathrm{~mL})$ was heated for $24 \mathrm{~h}$ at $50^{\circ} \mathrm{C}$, and concentrated. This deprotection procedure was repeated twice, and crude 36 was purified by column chromatography $\left(8: 2 \mathrm{CH}_{2} \mathrm{Cl}_{2}\right.$ :acetone, then $8: 2 \mathrm{CH}_{2} \mathrm{Cl}_{2}$ : methanol, both containing $1 \%$ triethylamine). To a solution of 36 in 1:2:2 EtOAc:2-propanol:methanol (2.5 $\mathrm{mL})$ was added $\mathrm{Pd} / \mathrm{C}(10 \mathrm{mg})$, and the mixture was hydrogenolysed at $4 \mathrm{~kg} / \mathrm{cm}^{2}$ for $24 \mathrm{~h}$. After filtration, the mixture was concentrated, and purified by Bio-Gel P-2 gel-permeation chromatography, affording $1(2.1 \mathrm{mg}, 75$ $\%)$ as a white powder. ${ }^{1} \mathrm{H}$ NMR $\left(\mathrm{D}_{2} \mathrm{O}\right) \delta 5.396(\mathrm{~d}, 1 \mathrm{H}$, H-1"'), 5.134 (d, 1H, H-1'), 5.125 (d, 1H, H-1"), 3.40 (m, $\left.2 \mathrm{H}, \quad \mathrm{O}\left(\mathrm{CH}_{2}\right)_{2} \mathrm{C} \mathrm{H}_{2} \mathrm{~N} \mathrm{D}_{2}\right), 2.82$ (brd, $2 \mathrm{H}$, $\left.\mathrm{OCH}_{2}\left(\mathrm{CH}_{2}\right)_{2} \mathrm{ND}_{2}\right), 2.027\left(\mathrm{~m}, 2 \mathrm{H}, \mathrm{OCH}_{2} \mathrm{CH}_{2} \mathrm{CH}_{2} \mathrm{ND}_{2}\right)$, $1.273\left(\mathrm{~d}, 3 \mathrm{H}, 3 \mathrm{H}-6^{\prime}\right) ; J_{1^{\prime}, 2^{\prime}}=1.8, J_{1^{\prime \prime}, 2^{\prime \prime}}=3.7, J_{1^{\prime \prime}, 2^{\prime \prime \prime}}=$ $3.7, J_{5^{\prime}, 6^{\prime}}=4.4 \mathrm{~Hz}$; Anal. MS (FAB) calcd for $\mathrm{C}_{26} \mathrm{H}_{50} \mathrm{NO}_{22} \mathrm{P}: 760.6\left(\mathrm{M}+\mathrm{H}^{+}\right)$; found $\mathrm{m} / \mathrm{z}$ : $760.6(\mathrm{M}+$ $\mathrm{H}^{+}$).

(Received in U.S.A. 8 March 1994; accepted 27 April 1994)

\section{Acknowledgments}

This investigation was supported with financial aid from the Institute of Molecular Biology and Medical Biotechnology (IMB, Utrecht University). The authors would like to thank Dr A. A. Bergwerff and Dr P. H. Kruiskamp for recording the NMR spectra of compound 1, and Mrs A. van der Kerk-van Hoof for recording the FAB-MS spectrum.

\section{References}

1. van Dam, J. E. G.; Fleer, A.; Snippe, H. Anthonie van Leeuwenhoek $1990,58,1$.

2. Robbins, J. B.; Austrian, R.; Lee, C.-J.; Rastogi, S. C.; Schiffman, G.; Henrichsen, J.; Mäkelä, P. H.; Broome, C. V.; Facklam, R. R.; Tiesjema, R. H.; Parke, Jr J. C. J. Infect. Dis. $1983,148,1136$.

3. Slaghek, T. M.; van Vliet, M. J.; Maas, A. A. M.; Kamerling. J. P.; Vliegenthart, J. F. G. Carbohydr. Res. 1989, 195, 75.

4. Slaghek, T. M.; van Oijen, A. H.; Maas, A. A. M.; Kamerling, J. P.; Vliegenthart, J. F. G. Carbohydr. Res. 1990, $207,237$.

5. Slaghek, T. M.; Maas, A. A. M.; Kamerling, J. P.; Vliegenthart, J. F. G. Carbohydr. Res. 1991, 211, 25.

6. Garegg, P. J.; Norberg, T. Carbohydr. Res. 1983, 116, 308.

7. Grzeszczyk, B.; Banaszek, A.; Zamojski, A. Carbohydr. Res. $1988,175,215$.

8. Kim, S.; Chang, H.; Kim, W. J. J. Org. Chem. 1985, 50, 1751.

9. Veeneman, G. H.; van Leeuwen, S. H.; van Boom, J. H. Tetrahedron Lett. 1990, 31, 1331.

10. van Boeckel, C. A. A.; Beetz, T. Tetrahedron Lett. 1983, 24,3775 .

11. Nilsson, S.; Lönn, H.; Norberg, T. Glycoconjugate J. 1999 , 6,21 .

12. Veeneman, G. H.; van Boom, J. H. Tetrahedron Lett. 1990 , $31,275$.

13. Fraser-Reid, B.; Wu, Z.; Andrews, C. W.; Skowronski, E. J. Am. Chem. Soc. 1991, 113, 1434.

14. Froehler, B. C.; Matteucci, M. D. Tetrahedron Lett. 1986, 27, 469.

15. van Steijn, A. M. P.; Jetten, M.; Kamerling, J. P.: Vliegenthart, J. F. G. Recl. Trav. Chim. Pays-Bas 1989, 108, 374.

16. Utamura, T.; Kuromatsu, K.; Suwa, K.; Koizumi, K.; Shingu, T. Chem. Pharm. Bull. 1986, 34, 2341. 